\title{
THE ITERATED PROJECTION METHOD FOR INTEGRO-DIFFERENTIAL EQUATIONS WITH CAUCHY KERNEL
}

\author{
ABDELAZIZ MENNOUNI
}

\begin{abstract}
In this paper we propose the iterated projection method for the approximate solution of an integro-differential equations with Cauchy kernel in $L^{2}([-1,1], \mathbb{C})$ using Legendre polynomials. We prove the convergence of the method. A system of linear equations is to be solved. Numerical examples illustrate the theoretical results.
\end{abstract}

AMS Mathematics Subject Classification : 45E05, 35J15.

Key words and phrases : Cauchy kernel, integro-differential equations, projection method.

\section{Introduction}

Many problems of mathematical physics such as unsteady aerodynamics and aero elastic phenomena, visco-elasticity, fluid dynamics, electrodynamics lead to a integro-differential equations with Cauchy kernel. Many integro-differential equations need to be solved numerically. Several authors have been studied projection approximations for solving integral equations with different numerical procedures, the theory of projection approximations is developed in [3]. In [10], the authors have discussed the superconvergence of the Galerkin iterates for integral equations of the second kind. In [6], we have studied projection approximations for solving Cauchy integro-differential equations using airfoil polynomials of the first kind. In [7], we have applied the successive approximation method, for solving a Cauchy singular integral equations of the first kind in the general case. In [5], we have introduced a new projection method, for solving integro-differential equations with Cauchy kernel. In the same perspective, we introduce a new application of the iterated projection method. The purpose of the present method is to approximate the solution of integro-differential equations with Cauchy Kernel using the iterated projection.

Received May 7, 2012. Revised November 23, 2012. Accepted February 15, 2013.

(c) 2013 Korean SIGCAM and KSCAM. 


\section{Main results}

Let the universe of our discours be the Hilbert space $\mathbb{H}:=L^{2}([-1,1], \mathbb{C})$. Set

$$
\mathbb{D}:=\left\{\varphi \in \mathbb{H}: \varphi^{\prime} \in \mathbb{H}, \varphi(-1)=0\right\},
$$

and consider the integro-differential equation with Cauchy kernel

$$
\varphi^{\prime}(s)+\oint_{-1}^{1} \frac{\varphi(t)}{t-s} d t=f(s), \quad-1 \leq s \leq 1,
$$

where the integral is understood as the Cauchy principal value:

$$
\oint_{-1}^{1} \frac{\varphi(t)}{t-s} d t=\lim _{\epsilon \rightarrow 0}\left(\int_{-1}^{s-\epsilon} \frac{x(t)}{t-s} d t+\int_{s+\epsilon}^{1} \frac{x(t)}{t-s} d t\right) .
$$

Letting

$$
\begin{aligned}
T x(s) & :=\oint_{-1}^{1} \frac{x(t)}{t-s} d t, \quad x \in \mathbb{H}, \quad-1 \leq s \leq 1, \\
A x(s) & :=x^{\prime}(s), \quad x \in \mathbb{D}, \quad-1 \leq s \leq 1,
\end{aligned}
$$

the operator $T$ is bounded from $\mathbb{H}$ into itself and

$$
A^{-1} y(s)=\int_{-1}^{s} y(t) d t, \quad y \in \mathbb{H}, \quad-1 \leq s \leq 1,
$$

is compact (cf.[4]). Equation (1) can be rewritten as

$$
\varphi+A^{-1} T \varphi=A^{-1} f .
$$

Let

$$
K:=A^{-1} T,
$$

since $A^{-1}$ is compact and $T$ is bounded, the operator $K$ is compact from $\mathbb{H}$ into itself. We assume that -1 is not an eigenvalue of $K$, and consider the approximate problem of finding $\varphi_{n} \in \mathbb{H}$ such that

$$
\varphi_{n}+K \pi_{n} \varphi_{n}=A^{-1} f .
$$

Clearly, if such a function exists, it belongs to $\mathbb{D}$. Let $\left(L_{n}\right)_{n \geq 0}$ be the sequence of Legendre polynomials and

$$
e_{j}:=\sqrt{\frac{2 j+1}{2}} L_{j},
$$

the corresponding normalized sequence. We consider the sequence of finite rank orthogonal projections $\left(\pi_{n}\right)_{n \geq 1}$ defined by

$$
\pi_{n} x:=\sum_{j=0}^{n-1}\left\langle x, e_{j}\right\rangle e_{j} .
$$

Applying the operator $\pi_{n}$ to both sides of equation (2) we get

$$
\pi_{n} \varphi_{n}+\pi_{n} K \pi_{n} \varphi_{n}=\pi_{n} A^{-1} f,
$$


or, equivalently,

$$
\sum_{j=0}^{n-1}\left\langle\varphi_{n}, e_{j}\right\rangle e_{j}+\sum_{j=0}^{n-1}\left\langle\varphi_{n}, e_{j}\right\rangle \pi_{n} K e_{j}=\sum_{j=0}^{n-1}\left\langle A^{-1} f, e_{j}\right\rangle e_{j},
$$

and performing the inner product with $e_{i}$ we get the following system:

$$
\left\langle\varphi_{n}, e_{i}\right\rangle+\sum_{j=0}^{n-1}\left\langle\varphi_{n}, e_{j}\right\rangle\left\langle\pi_{n} K e_{j}, e_{i}\right\rangle=\left\langle A^{-1} f, e_{i}\right\rangle, \quad i \in \llbracket 0, n-1 \rrbracket .
$$

Since $\pi_{n}^{*}=\pi_{n}$, and $\pi_{n} e_{i}=e_{i}$,

$$
\left\langle\varphi_{n}, e_{i}\right\rangle+\sum_{j=0}^{n-1}\left\langle\varphi_{n}, e_{j}\right\rangle\left\langle K e_{j}, e_{i}\right\rangle=\left\langle A^{-1} f, e_{i}\right\rangle, \quad i \in \llbracket 0, n-1 \rrbracket .
$$

Thus

$$
\left(I_{n}+A_{n}\right) x_{n}=b_{n},
$$

where $x_{n}(j):=\left\langle\varphi_{n}, e_{j}\right\rangle$, and

$$
\begin{aligned}
A_{n}(i, j) & :=\sqrt{\frac{2 j+1}{2}} \sqrt{\frac{2 i+1}{2}} \int_{-1}^{1} \int_{-1}^{s} \oint_{-1}^{1} \frac{L_{j}(\tau) L_{i}(s)}{\tau-t} d \tau d t d s, \\
b_{n}(i) & :=\sqrt{\frac{2 i+1}{2}} \int_{-1}^{1} \int_{-1}^{s} f(t) L_{i}(s) d t d s .
\end{aligned}
$$

Since $K$ is compact, $\left(I_{n}+A_{n}\right)^{-1}$ exists for $n$ large enough (see [3]). Once the system (3) is solved, $\varphi_{n}$ is recovered as

$$
\varphi_{n}(s)=\int_{-1}^{s} f(t) d t-\sum_{j=0}^{n-1} x_{n}(j) \sqrt{\frac{2 j+1}{2}} \int_{-1}^{s} \oint_{-1}^{1} \frac{L_{j}(\tau)}{\tau-t} d \tau d t .
$$

Let $s>0$ and $H^{s}([-1,1], \mathbb{C})$ be the classical Sobolev space, and let $\|\cdot\|_{s}$ denote its norm. (For details, see [2].) Remark that

$$
\left(I+A^{-1} T\right)\left(H^{s}([-1,1], \mathbb{C})\right)=H^{s}([-1,1], \mathbb{C}) .
$$

We recall that (cf. [2]) there exists $c>0$ such that, for all $\psi \in H^{s}([-1,1], \mathbb{C})$,

$$
\left\|\left(I-\pi_{n}\right) \psi\right\| \leq c n^{-s}\|\psi\|_{s} .
$$

Let

$$
M:=\sup _{n \geq N}\left\|\left(I+K \pi_{n}\right)^{-1}\right\|
$$

which is finite.

Theorem 2.1. Assume that $f \in H^{s}([-1,1], \mathbb{C})$ for some $s>0$. Then, there exists $\beta>0$ such that

$$
\left\|\varphi_{n}^{S}-\varphi\right\| \leq M \beta\|K\| n^{-s}\|\varphi\|_{s}
$$


TABLE 1. Absolute error for Example 1

\begin{tabular}{|c|c|}
\hline$n$ & $\left\|\varphi_{n}^{S}-\varphi\right\|$ \\
\hline 2 & $2.03 \mathrm{e}-1$ \\
4 & $2.15 \mathrm{e}-2$ \\
6 & $2.59 \mathrm{e}-3$ \\
8 & $3.44 \mathrm{e}-4$ \\
10 & $4.81 \mathrm{e}-5$ \\
12 & $6.98 \mathrm{e}-6$ \\
14 & $1.03 \mathrm{e}-6$ \\
16 & $1.57 \mathrm{e}-7$ \\
\hline
\end{tabular}

Proof. We have

$$
\begin{aligned}
\varphi_{n}^{S}-\varphi & =\left(A^{-1} f-K \pi_{n} \varphi_{n}^{S}\right)-\left(A^{-1} f-K \varphi\right) \\
& =K\left(I-\pi_{n}\right) \varphi+K \pi_{n}\left(\varphi-\varphi_{n}^{S}\right),
\end{aligned}
$$

and hence

$$
\left(\varphi_{n}^{S}-\varphi\right)=\left(I+K \pi_{n}\right)^{-1} K\left(I-\pi_{n}\right) \varphi .
$$

But $f \in H^{s}([-1,1], \mathbb{C})$, so $\varphi \in H^{s}([-1,1], \mathbb{C})$. Using (4), the desired result follows.

\section{Numerical Examples}

In this section, we present two numerical examples to illustrate the theoretical results obtained in the above section. Tables 1 and 2 show the absolute error as a function of $n$. We applied our method to solve (1) numerically. Once the system (3) is solved, we obtain the numerical results.

Example 1. We consider the integro-differential equation (1) with $f$ such that

$$
\begin{aligned}
f(s)= & -\frac{\pi s^{3}+(2-\pi) s^{2}+(4+\pi) s-2-\pi}{2\left(s^{2}+1\right)^{2}} \\
& -\frac{\left(2 s^{3}+2 s^{2}+2 x+2\right)}{\left(s^{2}+1\right)^{2}} \ln (s+1) \\
& +\frac{2 s^{3}+2 s^{2}+2 s+2}{\left(s^{2}+1\right)^{2}} \ln (1-s) .
\end{aligned}
$$

The exact solution be

$$
\varphi(s)=\frac{s+1}{s^{2}+1} .
$$


TABLE 2. Absolute error for Example 2

\begin{tabular}{|c||c|}
\hline$n$ & $\left\|\varphi_{n}^{S}-\varphi\right\|$ \\
\hline 6 & $8.11 \mathrm{e}-1$ \\
8 & $2.70 \mathrm{e}-1$ \\
10 & $7.94 \mathrm{e}-2$ \\
12 & $2.14 \mathrm{e}-2$ \\
14 & $5.07 \mathrm{e}-3$ \\
16 & $9.94 \mathrm{e}-4$ \\
18 & $1.16 \mathrm{e}-4$ \\
20 & $2.56 \mathrm{e}-5$ \\
22 & $3.96 \mathrm{e}-6$ \\
24 & $5.85 \mathrm{e}-7$ \\
\hline
\end{tabular}

Example 2. In this example we consider the integro-differential equation (1) with $f$ such that

$$
\begin{aligned}
f(s)=p(s) & -\left(s^{30}+6 s^{25}+15 s^{20}+20 s^{15}+15 s^{10}+6 s^{5}+1\right) \ln (s+1) \\
& +64 \ln (1-s)+960 \ln |s|
\end{aligned}
$$

where $p(s)$ is a known polynomial of degree 29 .

The exact solution be

$$
\varphi(s)=\left(s^{5}+1\right)^{6} .
$$

Example 3. Here we review the first example given in [6]. Let us consider the following integro-differential equation

$$
\varphi^{\prime}(s)+\frac{1}{\pi^{5}} \oint_{-1}^{1} \frac{\varphi(t)}{t-s} d t=\frac{2\left(1+\pi^{5}\right) s}{\pi^{5}}+\frac{\left(s^{2}-1\right)}{\pi^{5}} \ln \left(\frac{1-s}{1+s}\right)
$$

The exact solution is

$$
\varphi(s)=s^{2}-1 .
$$

Table (3) gives the numerical results for Example 3.

Example 4. We review the second example given in [6]. We consider the following integro-differential equation

$$
\varphi^{\prime}(s)+\oint_{-1}^{1} \frac{\varphi(t)}{t-s} d t=\frac{7}{3}-5 s^{2}+\left(s-s^{3}\right) \ln \left(\frac{1-s}{1+s}\right)
$$

The exact solution for this equation is

$$
\varphi(s)=-s^{3}+s .
$$

Table (4) gives the numerical results for Example 4. 
TABLE 3. Absolute error for Example 3

\begin{tabular}{|c|c|}
\hline$n$ & $\left\|\varphi_{n}^{S}-\varphi\right\|$ \\
\hline 3 & $2.07 \mathrm{e}-1$ \\
5 & $2.16 \mathrm{e}-2$ \\
7 & $2.63 \mathrm{e}-3$ \\
9 & $3.15 \mathrm{e}-4$ \\
11 & $4.56 \mathrm{e}-5$ \\
13 & $6.77 \mathrm{e}-6$ \\
\hline
\end{tabular}

TABLe 4. Absolute error for Example 4

\begin{tabular}{|c|c|}
\hline$n$ & $\left\|\varphi_{n}^{S}-\varphi\right\|$ \\
\hline 5 & $8.27 \mathrm{e}-1$ \\
7 & $2.36 \mathrm{e}-1$ \\
9 & $7.88 \mathrm{e}-2$ \\
13 & $2.09 \mathrm{e}-2$ \\
15 & $5.23 \mathrm{e}-3$ \\
17 & $9.89 \mathrm{e}-4$ \\
19 & $1.09 \mathrm{e}-4$ \\
\hline
\end{tabular}

\section{Conclusions}

Several projection methods have been studied extensively in the literature for approximating the solution of integral equations. In [9], the authors have presented a new approach to the numerical solution of integral equations. In [10], the authors have discussed the superconvergence of the Galerkin iterates for integral equations of the second kind. This work extends the application of the iterated projection method to singular integro-differential equations of Cauchy type. The present projection approximations based on Legendre polynomials. We have shown the convergence of the approximate solution to the exact solution in the Sobolev spaces. Numerical experiments show the pertinence of our method. The proposed method can be used to solve other class of integral and integro-differential equations.

\section{REFERENCES}

1. K. Atkinson, The Numerical Solution of Integral Equations of the Second Kind, Cambridge university press, 1997.

2. K.E. Atkinson, Han, Theoretical Numerical Analysis: A Functional Analysis Framework, 3rd edition Springer-Verlag, 2009.

3. K. Atkinson, The Numerical Solution of Integral Equations of the Second Kind, Cambridge university press, 1997.

4. H. Brezis, Analyse Fonctionnelle ,Dunod, Paris, 1999. 
5. A. Mennouni, A projection method for solving Cauchy singular integro-differential equations, Applied Mathematics Letters 25, (2012) 986-989.

6. A. Mennouni, S. Guedjiba, A note on solving integro-differential equation with Cauchy Kernel, Mathematical and Computer Modelling, 52 (2010) 1634-1638.

7. A. Mennouni, S. Guedjiba, A note on solving Cauchy integral equations of the first kind by iterations, Applied Mathematics and Computation, 217 (2011) 7442-7447.

8. G. M. Phillips, Interpolation and Approximation by Polynomials, Springer, 2003.

9. I. H. Sloan, B. J. Burn, N. Datyner, A new approach to the numerical solution of integral equations, J. Computational Phys., 18 (1975), 92105.

10. I.H. Sloan, V. Thomee, Superconvergence of the Galerkin iterates for integral equations of the second kind, J. Integral Equations, 9 (1985), 2-23.

Abdelaziz Mennouni is an associate professor in Department of Mathematics, and he received Ph.D in Applied Mathematics from Jean-Monnet University, France and from Biskra University, Algeria 2011. He received MAGISTER, functional analysis, from the University of M'sila Algeria 2005. He remained interested in: Applied mathematics, integral and integro-differential equations- singular integral equations- numerical Analysis, operators theory. He is potential reviewer in: Journal of Applied Mathematics and Computing - Scientific Research and Essays. He has published many papers in international refereed journals, and book chapter. He has also participated in many international conferences, recently, The Eleventh International Conference on Integral Methods in Science and Engineering, IMSE 2010, University of Brighton, UK.

Department of Mathematics, University of Mohamed el Bachir el Ibrahimi Bordj BouArreridj, Algeria.

e-mail: aziz.mennouni@yahoo.fr 